\title{
Comment naquit le Dictionnaire des lieux imaginaires
}

\section{Alberto Manguel}

Traducteur : Agnès Calza

\section{OpenEdition \\ Journals}

Édition électronique

URL : https://journals.openedition.org/rbnu/2874

DOI : $10.4000 /$ rbnu. 2874

ISSN : 2679-6104

\section{Éditeur}

Bibliothèque nationale et universitaire de Strasbourg

\section{Édition imprimée}

Date de publication : 1 mai 2012

Pagination : 82-83

ISSN : 2109-2761

\section{Référence électronique}

Alberto Manguel, "Comment naquit le Dictionnaire des lieux imaginaires », La Revue de la BNU [En ligne], 5 | 2012, mis en ligne le 01 mai 2012, consulté le 25 août 2021. URL : http:// journals.openedition.org/rbnu/2874; DOI : https://doi.org/10.4000/rbnu.2874

Ce document a été généré automatiquement le 25 août 2021.

\section{c) (i) (2)}

La Revue de la BNU est mise à disposition selon les termes de la Licence Creative Commons Attribution - Pas d'Utilisation Commerciale - Partage dans les Mêmes Conditions 4.0 International. 


\title{
Comment naquit le Dictionnaire des lieux imaginaires
}

\author{
Alberto Manguel \\ Traduction : Agnès Calza
}

Heureux le pays qui n'a pas de géographie ${ }^{1}$, Saki

(H. H. Munro)

1 Grâce à Google Earth, il est désormais possible de voir, sur son écran, chaque détail de notre planète. Pas seulement la grande sphère bleue que les satellites nous permettaient de voir de l'espace, corroborant l'intuition d'Éluard pour qui « la terre est bleue comme une orange»; pas seulement les masses continentales dérivant lentement, trop lentement pour être perceptibles à l'œil nu, et les lignes des fleuves semblables à des veines, et les lignes des chaînes de montagnes semblables à des cicatrices s'entrecroisant. Désormais, la technologie vous permet de voir les forêts et les vallées, les villes et les villages, les bâtiments des maisons et les arrière-cours. De l'autre bout du monde, vous pouvez presque apercevoir l'intérieur d'un salon à Tombouctou ou épier une réunion de famille aux Tonga. Nous avons rendu impossible la navigation vers l'inconnu telle que la pratiquait Ulysse. Le folle volo ${ }^{2}$ n'est plus possible, sauf sous surveillance humaine. Nous avons détruit l'intimité.

2 Jusqu'au siècle dernier, il était encore possible de s'imaginer des paysages pas encore décrits, dans quelques régions dispersées du monde qui n'avaient pas encore été explorées ou cartographiées. Sur le globe posé sur mon bureau d'enfant, il y avait des plaques roses, ici et là, que je trouvais bien plus attirantes que les pays officiellement délimités par des lignes et des pointillés, étiquetés par de grasses majuscules, avec leurs frontières politiques sévèrement tracées. Plutôt que d'accepter que cette partie fût la Roumanie et ce point précis Bucarest, je préférais inventer pour les plaques roses de l'Afrique et des pôles ma propre géographie, avec des noms plus mystérieux encore que le Tanganyika et des lieux plus séduisants que le lac Titicaca. Puis, quelques années plus tard seulement, on m'ôta cette liberté, et même les quelques endroits encore inconnus devinrent connus, et furent étiquetés à jamais. Mon exploration cessa, hormis dans le royaume sans danger du Guide bleu. 
3 Puis, vers le milieu des années 1970, je rencontrai Gianni Guadalupi. J'avais commencé à travailler pour Franco Maria Ricci à Milan, chez qui Gianni était rédacteur en chef, et nous devînmes rapidement amis. L'immense générosité intellectuelle de Gianni, son brillant sens de l'humour, son érudition tranquille m'attiraient, et bientôt nous devisâmes des moyens de détourner notre travail éditorial. Gianni était passionné d'ouvrages d'histoire farfelus (en particulier les histoires en « Et si », qui imaginaient ce qui serait arrivé si Napoléon avait gagné Waterloo ou si Hannibal n'avait pas été vaincu) et d'atlas. Bien qu'il ne fût pas amateur de vrais voyages, il se plaisait à suivre les sentiers et les routes tracés dans les vieilles cartes et les Baedeker, dont il avait une splendide collection, stockée dans un poulailler restauré à Arona, au plafond duquel il avait peint des fresques naïves représentant les sept merveilles du monde.

Dans les bureaux de Ricci, nous avons pleinement apprécié de travailler ensemble. Nous avons commandé (et parfois créé nous-mêmes) des textes pour plusieurs anthologies, nous avons traduit avec une licence plus que poétique toutes sortes de fictions et d'essais (moi de l'anglais vers l'espagnol, puis Gianni de l'espagnol vers l'italien), nous avons composé plusieurs numéros de la revue de Ricci et, ce qui nous enchantait pardessus tout, nous avons passé de longues heures à parler de livres et à lire.

Un jour, Gianni me parla d'un roman qu'il avait découvert, La ville vampire de Paul Féval, et dit que ce serait amusant d'écrire une sorte de guide touristique de la ville vampire, avec des informations sur les moyens de s'y rendre, les endroits où dormir, que manger, quels sites visiter - toutes ces informations étant extraites du roman luimême. Nous n'inventerions rien. Nous nous mîmes immédiatement au travail et composâmes bientôt quatre ou cinq pages d'un guide du voyageur dans la ville de Féval. Mais pourquoi s'en tenir là ? se demanda Gianni. Pourquoi ne pas étendre notre guide à d'autres villes imaginaires? Pourquoi ne pas y inclure des pays, ou même des continents? Nous avons commencé à dresser une liste des endroits imaginaires que nous connaissions. Bientôt, notre liste atteignit plusieurs centaines d'entrées. Voilà l'origine du Dictionnaire des lieux imaginaires.

6 Nous découvrîmes rapidement que la géographie de l'imaginaire était infiniment plus vaste que celle du monde réel. Cette affirmation, bien que banale, nous permit de percevoir l'immense générosité contenue dans la fonction vitale qui donne vie à des paysages et à des créatures qui ne peuvent prétendre à l'existence dans notre monde dense et pesant. Tels les anges dont nos ancêtres étudiaient la hiérarchie, telle la licorne et la manticore, tel l'indescriptible éther et le mystérieux phlogistique, telles les notions de démocratie parfaite et de bonne volonté pour tous les hommes, les lieux imaginaires que nous inventons n'ont besoin d'aucune incarnation pour exister dans notre esprit. L'Utopie et le Pays des merveilles, l'Atlantide et l'Eldorado sont toujours présents, bien qu'aucune carte officielle n'indique leur véritable localisation. «Elle n'est portée sur aucune carte: les vrais lieux n'y figurent jamais ${ }^{3}$, écrivait Herman Melville, après avoir visité une si grande partie du monde qu'on appelle réel. Gianni et moi étions bien d'accord.

7 Nous avons jugé nécessaire de restreindre notre recherche. Pour le bénéfice de l'économie du livre, nous avons éliminé paradis et enfers, ainsi que les endroits non situés sur terre. Nous avons choisi de ne pas y inclure les lieux imaginaires qui n'étaient que les pseudonymes de lieux réels, tels Yoknapatawpha County chez Faulkner ou le Balbec chez Proust. Nous avons décidé de ne pas explorer les mondes parallèles ou les lieux du futur, car (en accord avec la logique de notre Dictionnaire), ils entreraient en 
conflit ou empiéteraient sur les lieux de l'imaginaire « du présent ». Malgré tout, nous atteignîmes des milliers d'entrées. Bien sûr, des lieux imaginaires sont inventés chaque année, et notre Dictionnaire fut révisé et augmenté deux fois. À présent, il en est en réalité à sa troisième réincarnation.

Gianni est mort en 2005 mais sa "Wanderlust " littéraire hante encore les pages de notre Dictionnaire. En l'écrivant, il y a tant d'années, avec l'énergie et l'opiniâtreté que seule la jeunesse peut conjuguer, nous nous en sommes tenus strictement aux règles que nous nous étions fixées - rédiger les entrées comme si les lieux existaient réellement, et sans ajouter une seule information qui ne fût pas dans l'œuvre originale - avec deux exceptions. Nous avons décidé de nous autoriser à chacun l'invention d'un lieu, complété d'un auteur apocryphe et d'une bibliographie. Celui de Gianni était splendide : plein d'esprit, original et vraiment convaincant. Je ne révélerai pas quelles étaient ces deux « impostures ", mais j'ajouterai que lorsque le New York Times fit une critique du livre, le chroniqueur fit l'éloge de l'une de ces entrées, ajoutant qu'il était particulièrement heureux de cette insertion, car il avait lu le livre en question dans sa jeunesse et l'avait adoré, et qu'il ne l'avait jamais vu mentionné jusque-là.

Telle est la force de la fiction, en laquelle Gianni croyait avec tant d'intelligence.

\section{NOTES}

1. Traduction de Raymonde Weil et Michel Doury, in L'insupportable Bassington, suivi de Quatre nouvelles inédites, R. Laffont, 2006

2. Le «folle volo" fait référence à la Divine comédie de Dante, où Virgile et Dante rencontrent Ulysse dans le huitième cercle des Enfers. Ce dernier leur raconte que, contrairement à la légende homérique, il n'est pas rentré à Ithaque auprès de Pénélope. Motivé par l'exploration du monde et le goût de l'aventure, il parvint à convaincre son équipage de passer au-delà des colonnes d'Hercule, c'est-à-dire au-delà du monde connu. C'est ce que Dante appelle le "folle volo », le vol insensé. Mais passé ce cap, à la vue d'une terre nouvelle, Ulysse et son équipage font naufrage, mettant fin à ce « vol insensé » et à la volonté de connaissance qui guidait Ulysse (NDT).

3. Traduction d'Armel Guerne in Moby Dick, Phébus, 2005

\section{AUTEURS}

\section{ALBERTO MANGUEL}

Écrivain 